\title{
Atomically controlled quantum chains hosting a Tomonaga-Luttinger liquid
}

\author{
C. Blumenstein ${ }^{1}$, J. Schäfer ${ }^{1 \star}$, S. Mietke ${ }^{2}$, S. Meyer ${ }^{1}$, A. Dollinger ${ }^{1}$, M. Lochner ${ }^{1,2}$, X. Y. Cui ${ }^{3}$, \\ L. Patthey ${ }^{3}$, R. Matzdorf ${ }^{2}$ and R. Claessen ${ }^{1}$
}

\begin{abstract}
Atom assemblies on surfaces represent the ultimate lower size limit for electronic circuits, and their conduction properties are governed by quantum phenomena. A fundamental prediction for a line of atoms confining the electrons to one dimension is the Tomonaga-Luttinger liquid'. Yet, astonishingly, this has not been observed in surface systems so far. Here we scrutinize self-organized chains of single-atom width by scanning tunnelling spectroscopy and photoemission. The lowenergy spectra univocally show power-law behaviour. Even more, the density of states obeys universal scaling with energy and temperature. This demonstrates paradigmatic TomonagaLuttinger liquid properties ${ }^{2,3}$ encountered at the atomic scale, with bearing for the conductivity of wires and junctions. Local control enables us to study modified interactions due to defects or bridging atoms not previously possible.
\end{abstract}

Arrays of single atoms on surfaces provide an environment for a rich variety of quantum phenomena, especially regarding the electron states responsible for conduction. Their properties can be probed locally with scanning tunnelling microscopy (STM). Key examples include the superposition of electron waves in quantum corrals, leading to new coherent states ${ }^{4}$. A challenge remains the exotic correlated state predicted to occur when the electrons are squeezed into one dimension, as in a linear chain of atoms. Quantum theory describes this regime as a Tomonaga-Luttinger liquid $^{1}$ (TLL) with collective excitations of spin and charge. It reveals itself in characteristic power-law behaviour of the excitation spectra $^{2,3}$, with markedly depressed density of states at the chemical potential (where conduction takes place).

This state is highly fragile and collapses on slight coupling to the second dimension. Experimental indications of TLL low-energy spectra are scarce, consisting of one-dimensional (1D) crystals $^{5-7}$, carbon nanotubes ${ }^{8,9}$ and GaAs channels ${ }^{10,11}$ (compiled in Supplementary Table S1). Surprisingly, and contrary to expectations, this phenomenon has not been found so far in atom chains at surfaces, although such behaviour will dramatically affect atomic leads and junctions ${ }^{12}$.

Creation of a TLL state in such chains would be highly intriguing because this promises local atom manipulations that tune the interactions. Concerning approaches to build suitable chains, artificial atom placement by an STM tip ${ }^{13}$ leads to short arrays, which do not suffice for an extended 1D regime. Hence, our approach is to use self-organized chains of large extent formed by noble-metal atoms on the semiconducting $\mathrm{Ge}(001)$ surface $^{14,15}$. Here Au-induced chains show metallic tunnelling conductivity at room temperature ${ }^{15}$.

Self-organized formation of $\mathrm{Au}$ atom wires along the $\mathrm{Ge}(001)$ dimer rows leads to $c(8 \times 2)$ long-range order covering the
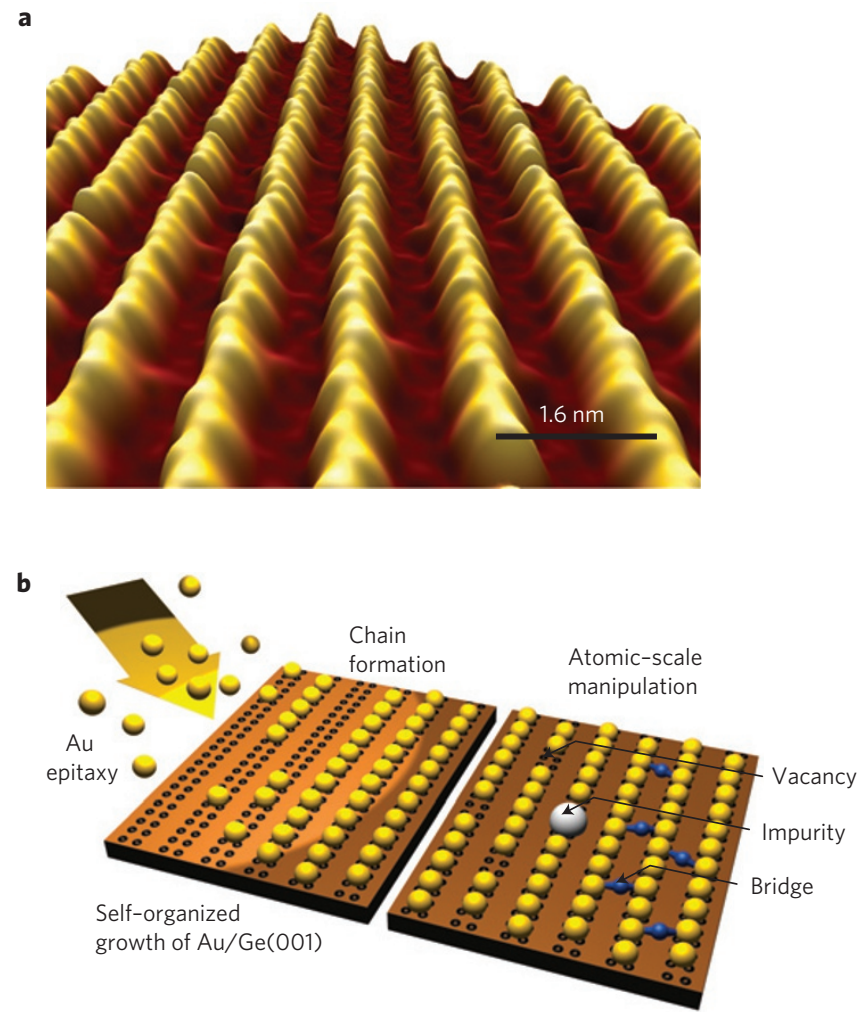

Figure 1 | Atomic arrangement of self-organized gold chains on $\mathrm{Ge}(001)$. a, Topography from STM, showing parallel alignment of Au-induced chains, spaced by $1.6 \mathrm{~nm}$ (bias $-1.0 \mathrm{~V}, 0.4 \mathrm{nA}$ ). The elevated chain architecture exposes a single-atom ridge, including a small buckling, which hosts the 1D electron liquid under study. $\mathbf{b}$, Schematic representation of chain formation and atomic modification possibilities. Epitaxy of Au leads to self-organized chain formation at elevated temperatures. Special atomic settings can be studied at vacancies (occurring randomly at subcritical coverage), leading to chain-end situations. These can also be realized by substrate terrace edges. Further modifications include impurity atoms (random or intentionally deposited), as well as bridges between chains (from additional atom deposition or placement with an STM tip). Such manipulations enable us to modify the electronic interactions in the chain

whole substrate ${ }^{15}$. The chains typically extend for several hundred nanometres. Aiming at spectroscopy of their electronic properties, this implies a level spacing well below $1 \mathrm{meV}$. The nanowires form elevated ridges with a line of single atoms on top, and a lateral

\footnotetext{
1Physikalisches Institut and Röntgen Research Center for Complex Material Systems, Universität Würzburg, Am Hubland, 97074 Würzburg, Germany, ${ }^{2}$ Institut für Physik, Universität Kassel, 34132 Kassel, Germany, ${ }^{3}$ Swiss Light Source, Paul-Scherrer-Institut, 5232 Villigen, Switzerland. ^e-mail: joerg.schaefer@physik.uni-wuerzburg.de.
} 
chain spacing of $1.6 \mathrm{~nm}$, as seen by STM in Fig. 1a. With such large separation, the chain architecture is well suited to host a 1D electron system with negligible interchain coupling. This is confirmed by angle-resolved photoemission, where we find a 1D electron pocket without any detectable dispersion perpendicular to the wire direction (see Supplementary Information SII). Therefore, the $\mathrm{Au}$ chains mark a structurally and electronically unusually well-defined 1D system.

Previously, nanowires were prepared by In atoms on $\mathrm{Si}(111)$ (ref. 16) and by $\mathrm{Au}$ atoms on $\mathrm{Si}(557)$ (ref. 17) or $\mathrm{Si}(553)$ (ref. 18). All these systems exhibit a lattice distortion below $\sim 150-200 \mathrm{~K}$, presumably a Peierls instability. This is indicative of lateral coupling that stabilizes the distortion, yet is detrimental to TLL physics. A claim of TLL behaviour in $\mathrm{Au} / \mathrm{Si}(557)$ (ref. 17) could not be maintained $^{19,20}$, and was explained by conventional band structure. Moreover, a Peierls instability implies pseudogaps ${ }^{21}$, which can obscure TLL low-energy spectra. In contrast, the $\mathrm{Au} / \mathrm{Ge}(001)$ chains are stable against a Peierls distortion even at liquid He temperature.

As a specific feature of our substrate-supported atom chains, different local scenarios may be realized as in Fig. 1b. Vacancy defects or substrate terrace edges provide a well-defined setting to study electron states in the vicinity of a chain end. Moreover, we may probe the environment of impurity atoms, which act as scatterers for the electrons, or even bridge atoms contacting two wires. The corresponding excitation spectra are accessed, spatially resolved, by scanning tunnelling spectroscopy (STS).

The differential tunnelling conductivity $\mathrm{d} I / \mathrm{d} V$ recorded on extended nanowires at $4.7 \mathrm{~K}$ is shown in Fig. 2a. This property is a good measure of the electronic density of states (DOS), because variations of the tunnelling-matrix element are negligible for the small voltages used. To average out effects from local orbitals on both the nanowire DOS and the matrix element, the data are integrated over typically $10^{3}$ spectra taken over several unit cells. Most notably, in an energy window from $-50 \mathrm{meV}$ to $+50 \mathrm{meV}$, we find a pronounced depression of the signal with a minimum at the chemical potential $\mu$.

The observed spectral behaviour seems to be consistent with a power-law dependence on energy. This is the prediction for a TLL, with its DOS $\sim E^{\alpha}$ (at $T=0$; refs 2,22). Here $\alpha$ is a non-universal exponent that is characteristic of the electron interaction in the chains. Specifically, a very close fit to the data is achieved for $\alpha=0.53$ (see Fig. 2a). This value has been confirmed for a variety of different tunnelling set points and temperatures within an error margin of \pm 0.05 (see Supplementary Information SIII). The fit contains a correction for the small but finite temperature, which affects both the spectral function of the electrons and the Fermi distribution of the tunnelling tip ${ }^{23}$.

An alternative explanation for a suppression of spectral weight in low-dimensional metals would be a Coulomb pseudogap, which occurs in the presence of disorder ${ }^{24,25}$. The functional form of this zero-bias anomaly, however, for low energies is described by an exponential decrease of the signal. The result of such a model, using the interaction strength as a free fit parameter, is included in Fig. 2a. Whereas at higher binding energies a coarse approximation to the data can be achieved, the match is increasingly poor at lower energies around $E=0$. Hence, a Coulomb pseudogap cannot explain the observed spectral behaviour.

We might furthermore consider a 'dynamical Coulomb blockade' due to the external impedance of the tunnelling circuit. For very high impedances this can also yield a power-law behaviour ${ }^{26}$. However, this effect only becomes noticeable at resistance values $R$ far above the resistance quantum $R_{\mathrm{Q}}=h / e^{2}=$ $26 \mathrm{k} \Omega$. In contrast, our experimental resistance is three orders of magnitude lower, so this regime definitely does not apply. Moreover, in that model the exponent $\alpha=2 R / R_{\mathrm{Q}}$ depends on the local impedance, with a resulting value of effectively zero (see
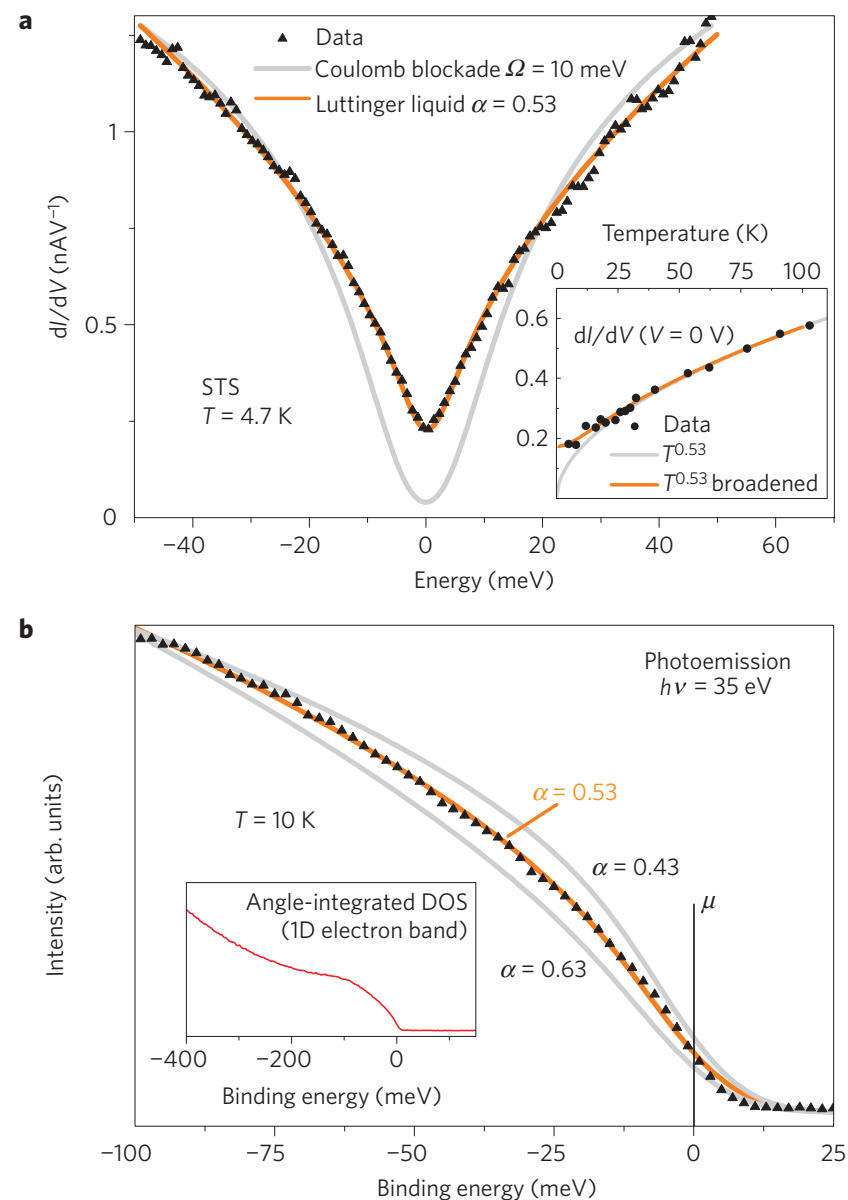

Figure $\mathbf{2}$ | Spectroscopy of the DOS at low energies. a, Differential conductivity from tunnelling spectroscopy. STS data at $4.7 \mathrm{~K}$ (averaged over $\sim 10^{3}$ spectra in a $30 \mathrm{~nm} \times 30 \mathrm{~nm}$ area to average out local atomic effects), showing a deep recess around zero bias. Orange curve, TLL model, a power law with exponent $\alpha=0.53$ (including the $T$-correction discussed in the text); grey, model for Coulomb blockade, fitted to data, yet providing no close match. Inset: $T$-dependent DOS at zero bias plotted up to $102 \mathrm{~K}$.

The data are described by a power law with the same exponent (grey); the orange curve includes experimental broadening. b, Photoemission spectrum of the 1D states at $10 \mathrm{~K}$ (angle-integrated over a 1D electron band to reflect the DOS). The data are fitted with a power law convolved with the experimental resolution. The exponent amounts to $\alpha=0.53$ with \pm 0.05 accuracy, thereby fully confirming the STS result as an intrinsic spectral function.

Supplementary Information SIV), which is not observed in our data. Hence, such a scenario can safely be excluded.

Importantly, there is further evidence from a different experiment that we measure intrinsic TLL properties. It is based on high-resolution photoemission, where no external voltage is used and a circuit-like situation as in conductance measurements does not apply. Photoemission spectra thus directly reflect the manybody spectral function ${ }^{22,23}$. The result in Fig. $2 \mathrm{~b}$ for the DOS of the $1 \mathrm{D}$ electron band ( $k$ integrated, for better comparison to the STS data) shows a collapse of the spectral weight, which can be fitted independently by a power law with $\alpha=0.53 \pm 0.05$. This fully coincides with the STS results, so the latter must be interpreted as an intrinsic spectral function of the electron liquid.

Further support for a TLL interpretation of the tunnelling spectra comes from their temperature dependence. We observe that the spectral dip around zero bias becomes filled with increasing temperature. The temperature dependence of the tunnelling DOS at 

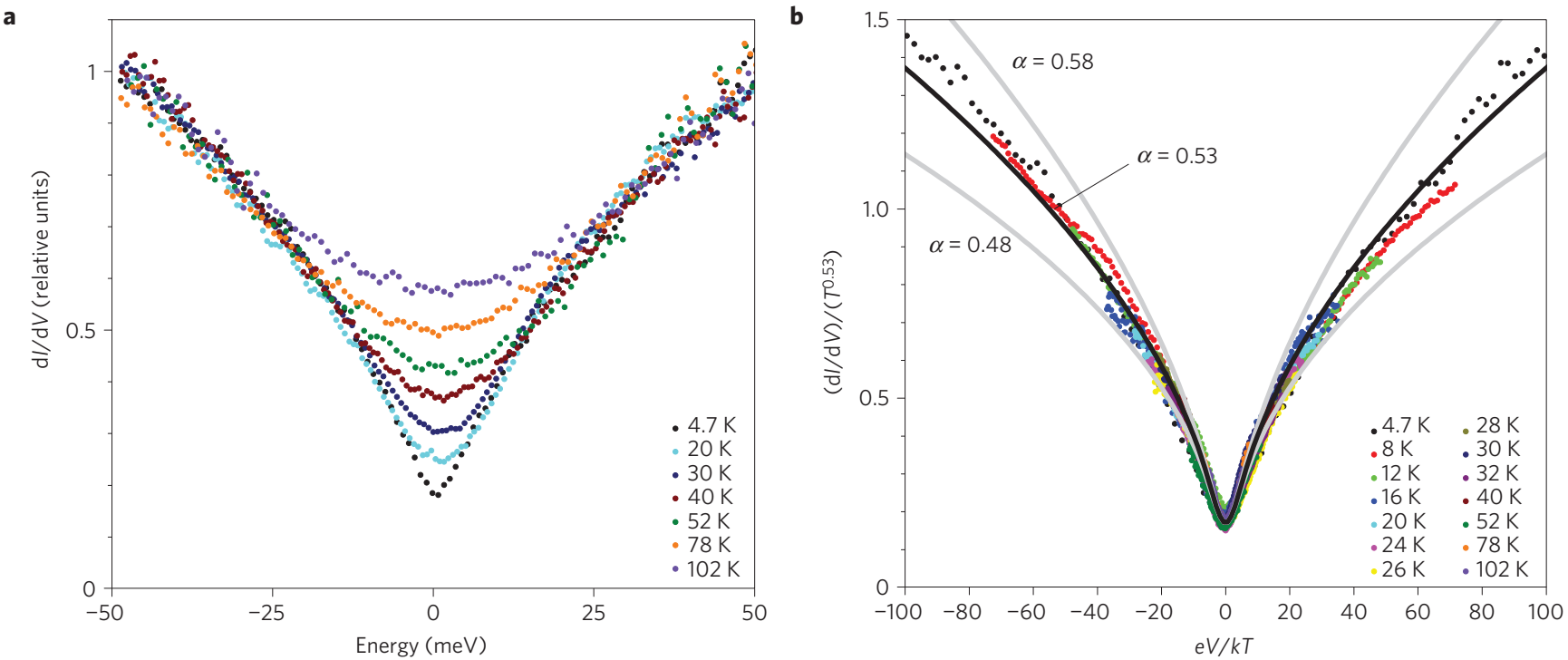

Figure 3 | Spectral properties investigated for a wide temperature range. a, Differential conductivity for a series of temperatures as indicated. The DOS at zero bias increases with $T$. b. Universal scaling plot of the spectral data, using a $T$-renormalized energy scale $e V / k T$. All curves coincide, showing universal scaling behaviour predicted as a hallmark of TLL behaviour. The fit curve accounts for thermal broadening in the TLL description as in equation (1), the $T$ dependence of the Fermi distribution for the tip, and is convoluted with the experimental resolution. Black curve: TLL power law with $\alpha=0.53$. Grey: for \pm 0.05 variation of $\alpha$ to test statistical confidence (for log-log plots see Supplementary Information SIII).

$E=0$ is depicted in the inset of Fig. 2a, with corresponding spectra shown in Fig. 3a. In TLL theory it is predicted to follow a power law for temperature with the same exponent as for energy ${ }^{23}$. In the inset of Fig. 2a we have overlaid a $T^{\alpha}$ curve with $\alpha=0.53$ (with experimental broadening). The theoretical curve yields a perfect fit of the data for the entire temperature range between 4.7 and $102 \mathrm{~K}$.

This identical power-law dependence in both energy and temperature is a key feature of TLL theory and results from the fact that the electronic low-energy excitation spectrum has no intrinsic energy scale other than the temperature ${ }^{2,3}$. Consequently, the tunnelling DOS follows a universal scaling relation ${ }^{23}$ as a function of $E$ and $T$, which can be written as ${ }^{8}$

$$
\operatorname{DOS}(E, T) \propto T^{\alpha} \cosh \left(\frac{E}{2 k T}\right)\left|\Gamma\left(\frac{1+\alpha}{2}+i \frac{E}{2 \pi k T}\right)\right|^{2}
$$

(where $\Gamma$ is the gamma function); that is, apart from the leading power-law factor the DOS depends only on the ratio $E / k T$.

The scaling behaviour can be directly tested by our tunnelling spectra. In Fig. $3 \mathrm{~b}$ the experimental $\mathrm{d} I / \mathrm{d} V$ curves of Fig. $3 \mathrm{a}$ are normalized to $T^{0.53}$ and plotted versus $\mathrm{eV} / \mathrm{kT}$. Very strikingly, all experimental spectra collapse onto a single curve as predicted by TLL theory. Furthermore, they strictly follow the theoretical behaviour (Fig. 3b), which results from a convolution of the scaling function (1) with the energy derivative of the Fermi-Dirac distribution function ${ }^{6,8}$. These observations thus provide critical proof for the TLL nature of the conduction-electron system in these $\mathrm{Au}$ atom chains.

The power-law exponent can be fixed with high accuracy to $\alpha=0.53$ (Fig. $3 \mathrm{~b}$ ). It is directly related to the so-called charge stiffness constant $K_{\rho}$ by

$$
\alpha_{\text {bulk }}=\left(K_{\rho}+K_{\rho}^{-1}-2\right) / 4
$$

with $K_{\rho}$ depending on the interaction strength in the 1D electron system (assuming repulsive and spin-rotation-invariant interaction $)^{2,3}$. This parameter controls also the behaviour of other electronic response functions (for example optical excitations) as well as that of the spatial spin or charge correlations. It is bound to a range between 0 and 1 , with $K_{\rho}=1$ (that is, $\alpha=0$ ) corresponding to the non-interacting limit. For the Au chains we obtain a value of $K_{\rho}=0.26$, which signals a fairly strong electronic interaction. This result compares well to the findings in bulk 1D systems. For example, a recent STS study ${ }^{6}$ on $\mathrm{Li}_{0.9} \mathrm{Mo}_{6} \mathrm{O}_{17}$ found $K_{\rho}=0.24$ (from $\alpha=0.62$ ), whereas for 1D gated semiconductor field-effect structures values of $K_{\rho}=0.26-0.28(\alpha=0.47-0.52)$ have been reported ${ }^{11}$.

A remarkable property of this surface-defined atomic system is the ability to study special configurations, such as a chain terminated on one side. This is, for example, realized by a surface terrace step of the $\mathrm{Ge}(001)$ substrate as in Fig. $4 \mathrm{a}$. In extending the TLL description to bounded electron liquids in semi-infinite chains $^{23,27}$, a different exponent $\alpha$ at the chain end is obtained as

$$
\alpha_{\text {end }}=\left(K_{\rho}^{-1}-1\right) / 2
$$

In using the above derived $K_{\rho}=0.26$, a theoretical $\alpha_{\text {end }}=1.43$ is predicted, notably increased over the chain bulk.

The experimental DOS spectrum for terminated chains in Fig. $4 \mathrm{~b}$ can be fitted with the $T$-corrected expression (1). Clearly, the apparent experimental exponent $\alpha=1.20$ is significantly enhanced over the bulk value in long chains, showing a strong effect of the edge singularity. It is still marginally smaller than the theoretical prediction $\alpha_{\text {end }}=1.43$ in (3). This can be understood when addressing the corresponding energy scales on which the end and bulk exponents govern the spectra. TLL theory predicts ${ }^{28}$ that the 'end spectrum' characterized by $\alpha_{\text {end }}$ exists up to a crossover energy $\omega_{\mathrm{c}}$, which collapses with distance $r$ from the edge as $\omega_{\mathrm{c}}(r) \sim 1 / r$. At energies above $\omega_{\mathrm{c}}$, the spectral shape is characterized by $\alpha_{\text {bulk }}$ as in equation (2). In the experiment close to the edge this implies that the finite area used to improve statistics will average over slightly different spectral shapes. It yields an 'apparent' exponent that is slightly less than the underlying TLL exponent $\alpha_{\text {end }}$.

In going further away from the chain end, the bulk exponent should start to dominate the spectrum, whereas the end-spectrum energy scale almost vanishes. This is indeed seen in the experimental data in Fig. 4c, where the apparent exponent is determined as $\alpha=$ 0.61 . It is already close to (but still slightly higher than) $\alpha_{\text {bulk }}=0.53$. 

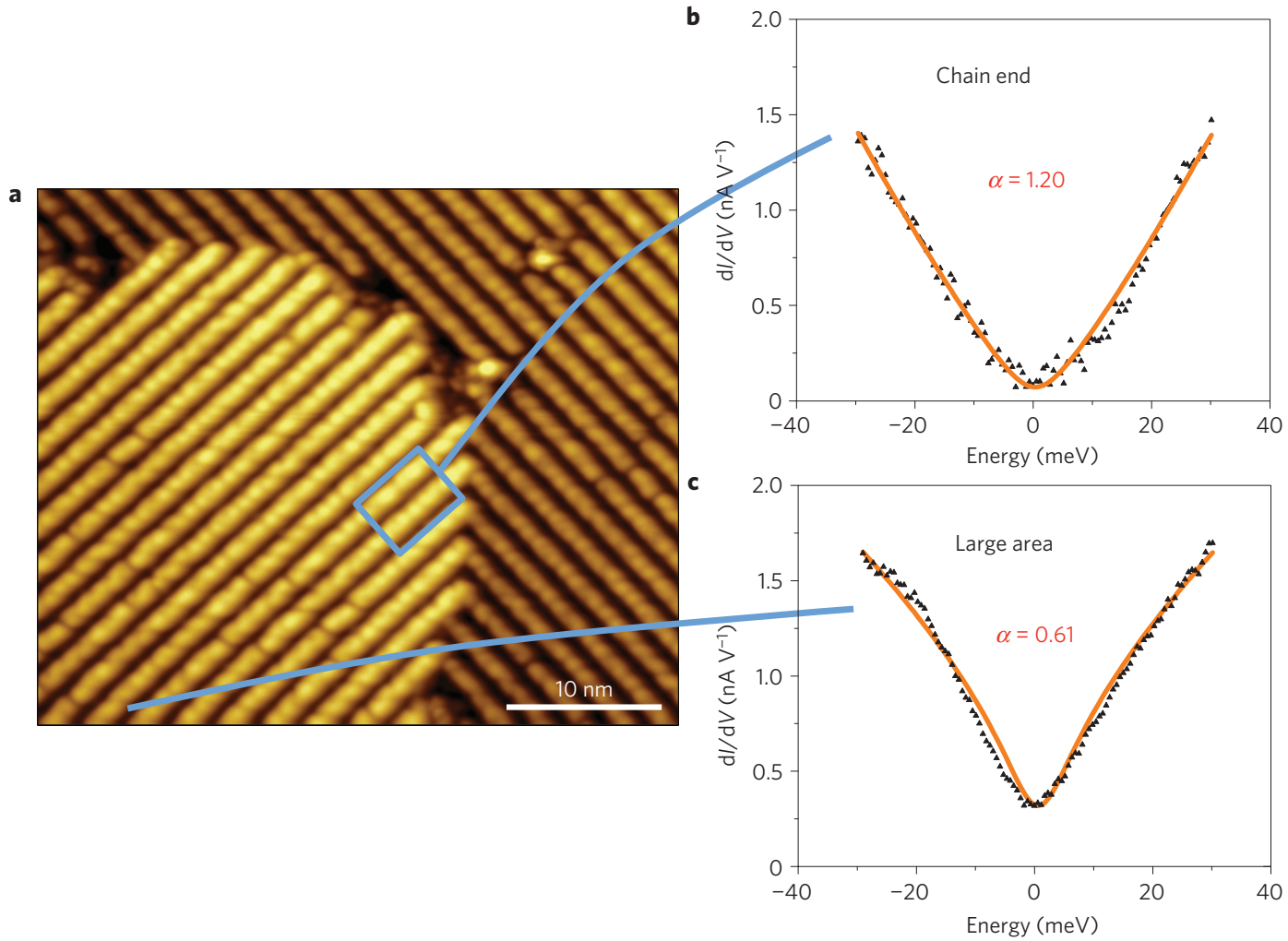

Figure 4 | Tunnelling spectra near chain end with increased exponent. a, STM of a terrace edge where a bundle of chains is terminated. Terraces on $\mathrm{Ge}(001)$ induce a $90^{\circ}$ rotation of the wire orientation. Chain ends can be directly accessed with the STM tip for spectroscopy. $\mathbf{b}, \mathrm{STS}$ data ( $T=4.7 \mathrm{~K}$ ) taken very close to the edge in the indicated area (averaged over two chains for statistics). The exponent is increased to an apparent value of $\alpha=1.20$, which is marginally smaller than the $\alpha_{\text {end }}=1.43$ predicted by TLL theory. The remaining discrepancy is ascribed to the rapid spatial collapse of the end-spectrum energy scale, which implies an experimental averaging effect. c, Further away from the edge (in a corresponding window), the spectrum has an apparent exponent $\alpha=0.61$, which in turn is dominated by the bulk value $\alpha_{\text {bulk }}=0.53$ (error bar for apparent exponents \pm 0.1 determined from the standard deviation).

With respect to local interactions, these nanowires offer the opportunity to locally scan the exponent of semi-infinite chains terminated by a vacancy, so that we may test predictions for spatial oscillations of the DOS (ref. 28). Moreover, the system can be modified by additional bridge atoms deposited between the chains, so that the induced interchain coupling provokes a 'dimensional crossover $^{29}$. This is highly relevant for any junction ${ }^{12}$ at the base of electronic circuits to be constructed with atoms or molecules. Thus, the TLL state now revealed has bearing for atomic wires and contacts at surfaces, and implies that such strong electron correlations significantly modify the conduction properties at the atomic scale.

\section{Methods}

The atomic chain growth was implemented under ultrahigh-vacuum conditions. $\mathrm{Ge}(001)$ substrates with moderate $\mathrm{n}$-doping $(0.4 \Omega \mathrm{cm})$ were used to ensure electron replenishment during spectroscopy measurements. They were chemically etched and oxide-desorbed in situ. Gold epitaxy conducted at a substrate temperature of $\sim 500^{\circ} \mathrm{C}$ provides the activation energy for self-assembly of the $\mathrm{Au}$ chain $s^{15}$. The optimum Au coverage $(\sim 0.5$ monolayers $)$ was judged by the $c(8 \times 2)$ pattern in electron diffraction.

STM and STS measurements were carried out with a low-temperature instrument with $\mathrm{He}$ cooling (Omicron), reaching a minimum temperature of $\sim 4.7 \mathrm{~K}$. Spectroscopy data were obtained from numerical differentiation of $I(\mathrm{~V})$ spectra. Set points of typically $0.05-0.5 \mathrm{nA}$ at $100 \mathrm{mV}$ bias were used, and the experimental resolution amounted to $5 \mathrm{meV}$. Tunnelling tips were made of tungsten and tested on a $\mathrm{Ag}(111)$ crystal to be devoid of tip-related spectral structures in the low-bias region.

Angle-resolved photoelectron spectroscopy was carried out at the Swiss Light Source at the Surface and Interface Spectroscopy beamline using the High-Resolution Photoelectron Spectroscopy endstation at $10 \mathrm{~K}$, providing a total resolution of $10 \mathrm{meV}$. The band situation with two electron pockets was noted by us earlier ${ }^{15}$ on the basis of He I excitation. Synchrotron $k$-space mapping of the
Fermi surface confirms that the electron system is of exceptional 1D character ${ }^{30}$. For interpretation as DOS to be compared with TLL theory, the spectra have been angle integrated over the occupied $k$ range.

Received 15 June 2010; accepted 22 June 2011; published online 7 August 2011

\section{References}

1. Haldane, F. D. M. 'Luttinger liquid theory' of one-dimensional quantum fluids: I. Properties of the Luttinger model and their extension to the general 1D interacting spinless Fermi gas. J. Phys. C 14, 2585-2609 (1981).

2. Voit, J. One-dimensional Fermi liquids. Rep. Prog. Phys. 58, 977-1116 (1995).

3. Giamarchi, T. Quantum Physics in One Dimension (Clarendon Press, 2003).

4. Manoharan, H. C., Lutz, C. P. \& Eigler, D. M. Quantum mirages formed by coherent projection of electronic structure. Nature 403, 512-515 (2000).

5. Claessen, R. et al. Spectroscopic signatures of spin-charge separation in the quasi-one-dimensional organic conductor TTF-TCNQ. Phys. Rev. Lett. 88, 096402 (2002).

6. Hager, J. et al. Non-Fermi-liquid behavior in quasi-one-dimensional $\mathrm{Li}_{0.9} \mathrm{Mo}_{6} \mathrm{O}_{17}$. Phys. Rev. Lett. 95, 186402 (2005).

7. Wang, F. et al. New Luttinger-liquid physics from photoemission on $\mathrm{Li}_{0.9} \mathrm{Mo}_{6} \mathrm{O}_{17}$. Phys. Rev. Lett. 96, 196403 (2006).

8. Bockrath, M. et al. Luttinger-liquid behaviour in carbon nanotubes. Nature 397, 598-601 (1999).

9. Ishii, H. et al. Direct observation of Tomonaga-Luttinger-liquid state in carbon nanotubes at low temperatures. Nature 426, 540-544 (2003).

10. Auslaender, O. M. et al. Spin-charge separation and localization in one dimension. Science 308, 88-92 (2005).

11. Jompol, Y. et al. Probing spin-charge separation in a Tomonaga-Luttinger liquid. Science 325, 597-601 (2009).

12. Nazin, G. V., Qiu, X. H. \& Ho, W. Visualization and spectroscopy of a metal-molecule-metal bridge. Science 302, 77-81 (2003).

13. Nilius, N., Wallis, T. M. \& Ho, W. Development of one-dimensional band structure in artificial gold chains. Science 297, 1853-1856 (2002). 
14. Stekolnikov, A. A., Bechstedt, F., Wisniewski, M., Schäfer, J. \& Claessen, R. Atomic nanowires on the Pt/Ge(001) surface: Buried Pt-Ge versus top Pt-Pt chains. Phys. Rev. Lett. 100, 196101 (2008).

15. Schäfer, J., Blumenstein, C., Meyer, S., Wisniewski, M. \& Claessen, R. New model system for a one-dimensional electron liquid: Self-organized atomic gold chains on Ge(001). Phys. Rev. Lett. 101, 236802 (2008).

16. Yeom, H. W. et al. Instability and charge density wave of metallic quantum chains on a silicon surface. Phys. Rev. Lett. 82, 4898-4901 (1999).

17. Segovia, P., Purdie, D., Hengsberger, M. \& Baer, Y. Observation of spin and charge collective modes in one-dimensional metallic chains. Nature 402, 504-507 (1999).

18. Ahn, J. R., Kang, P. G., Ryang, K. D. \& Yeom, H. W. Coexistence of two different Peierls distortions within an atomic scale wire: Si(553)-Au. Phys. Rev. Lett. 95, 196402 (2005).

19. Losio, R. et al. Band splitting for $\mathrm{Si}(557)-\mathrm{Au}$ : Is it spin-charge separation? Phys. Rev. Lett. 86, 4632-4635 (2001)

20. Ahn, J. R., Yeom, H. W., Yoon, H. S. \& Lyo, I-W. Metal-insulator transition in Au atomic chains on Si with two proximal bands. Phys. Rev. Lett. 91, 196403 (2003).

21. Schäfer, J. et al. Unusual spectral behavior of charge-density waves with imperfect nesting in a quasi-one-dimensional metal. Phys. Rev. Lett. 91, 066401 (2003).

22. Meden, V. \& Schönhammer, K. Spectral functions for the Tomonaga-Luttinger model. Phys. Rev. B 46, 15753-15760 (1992).

23. Schönhammer, K. \& Meden, V. Correlation effects in photoemission from low dimensional metals. J. Electron. Spectrosc. 62, 225-236 (1993).

24. Mishchenko, E. G., Andreev, A. V. \& Glazman, L. I. Zero-bias anomaly in disordered wires. Phys. Rev. Lett. 87, 246801 (2001).

25. Bartosch, L. \& Kopietz, P. Zero bias anomaly in the density of states of low-dimensional metals. Eur. Phys. J. B 28, 29-36 (2002).
26. Devoret, M. H. et al. Effect of the electromagnetic environment on the Coulomb blockade in ultrasmall tunnel junctions. Phys. Rev. Lett. 64, 1824-1827 (1990).

27. Kane, C. L. \& Fisher, M. P. A. Transport in a one-channel Luttinger liquid. Phys. Rev. Lett. 68, 1220-1223 (1992).

28. Eggert, S., Johannesson, H. \& Mattsson, A. Boundary effects on spectral properties of interacting electrons in one dimension. Phys. Rev. Lett. 76, 1505-1508 (1996).

29. Schulz, H. J. Long-range Coulomb interactions in quasi-one-dimensional conductors. J. Phys. C 16, 6769-6787 (1983).

30. Meyer, S. et al. Strictly one-dimensional electron system in Au chains on $\mathrm{Ge}(001)$ revealed by photoelectron $k$-space mapping. Phys. Rev. B 83, 121411(R) (2011).

\section{Acknowledgements}

We thank S. Eggert, V. Meden, B. Trauzettel, P. Recher and F. F. Assaad for theoretical discussions. We also acknowledge support by the Deutsche Forschungsgemeinschaft under grants Scha 1510/2 and FOR 1162.

\section{Author contributions}

C.B., J.S., S. Mietke, S. Meyer, A.D. and M.L. carried out the experiments, R.M. provided STS expertise, X.Y.C. and L.P. ran the photoemission station, C.B., J.S., S. Mietke, S. Meyer and A.D. analysed the data, C.B., J.S. and S. Meyer made the figures, J.S. and R.C. wrote the text and all authors contributed to critical discussion of the data.

\section{Additional information}

The authors declare no competing financial interests. Supplementary information accompanies this paper on www.nature.com/naturephysics. Reprints and permissions information is available online at http://www.nature.com/reprints. Correspondence and requests for materials should be addressed to J.S. 